\title{
Blood transfusion practice in surgery at Bugando Medical Centre in northwestern Tanzania
}

\author{
PHILLIPO L. CHALYA ${ }^{1 *}$, FIDELIS MBUNDA ${ }^{1}$, JOSEPH B. MABULA ${ }^{1}$, ANTONY N. MASSINDE ${ }^{2}$, ALBERT \\ KIHUNRWA ${ }^{2}$ and JAPHET M. GILYOMA ${ }^{1}$ \\ ${ }^{1}$ Department of Surgery, Catholic University of Health and Allied Sciences- Bugando, Mwanza, Tanzania \\ ${ }^{2}$ Department of Obstetrics \& Gynaecology, Catholic University of Health and Allied Sciences-Bugando, Mwanza, \\ Tanzania
}

\begin{abstract}
Background: Preoperative over-ordering of blood for surgical intervention, in excess of the actual and anticipated needs is a common practice in many developing countries. This can be decreased by simple means of changing the blood cross matching and ordering schedule depending upon the type of surgery performed. The aim of this study was to assess the blood transfusion practice in surgery at Bugando Medical Centre in northwestern Tanzania.

Methods: This was a retrospective study among patients undergoing major operations at Bugando Medical Centre. We evaluated blood ordering and transfusion practices in emergency and elective surgical procedures and calculated different indices such as cross-match to transfusion ratio ( $\mathrm{C} / \mathrm{T}$ ratio), transfusion probability (\% T) and transfusion index (TI). Next Maximal Surgical Blood Ordering System (MSBOS) was estimated for each procedure.

Results: The overall blood utilization was only $28.2 \%$, consisting of $17.1 \%$ in the elective operations and $26.9 \%$ in the emergency operations. There was no blood utilization for most of the routine elective cases suggesting cross-matching of blood to be a culture than necessity. Generally, the overall blood transfusion of the requested blood as indicated by indices of C/T ratio, \%T, TI and MSBOS were 3.5, 28.7\%, 0.33 and 0.45, respectively. The overall CT ratio, \%T, TI and MSBOS in the elective operations were 5.8, 15.9\%, 0.2 and 0.3, respectively. In the emergency operations, the overall $\mathrm{CT}$ ratio, \%, $\mathrm{TI}$ and MSBOS were $3.7,22 \%, 0.32$ and 0.48 , respectively.

Conclusion: This study demonstrated that over-ordering of blood in excess of the actual needs is a common practice at BMC. Blood ordering pattern needs to be revised and over-ordering of blood should be minimized. This can be possible by the estimation of MSBOS for each procedure and requisition as calculated.

Keywords: Blood transfusions, practice, surgery, Tanzania
\end{abstract}

\section{Introduction}

Blood transfusion plays a major role in the resuscitation and management of surgical patients and ordering of blood is usually a common practice in elective and emergency surgical procedures (Rund et al., 1992; Vibhute et al., 2000). The World Health Organization reports large differences in the amount of blood collected and transfused worldwide. Annual rates of using blood are 45.4 units per 1,000 population in high income countries, 10.1 units per 1,000 population in middle income countries, and 3.6 units per 1,000 population in low income countries (Hebert et al., 1999). Since the introduction of blood transfusion into clinical practice, its appropriate use has been the subject for debate. It has been reported that only $30 \%$ of cross-matched blood is used in elective surgery (Sowayan, 1994). In addition, a number of studies in many countries have shown over-ordering of blood by surgeons with utilization ranging from 5 to 40\% (Friedman et al., 1976).

\footnotetext{
*Correspondence: Dr. Phillipo L. Chalya; E-mail: drphillipoleo@yahoo.com
} 
In South Africa, for example, 7-10\% of blood is wasted annually because of over-ordering of blood (Efraim, 2001). Reports from India, Kuwait and Nigeria also show utilization rate of $28 \%$ (Vibhute et al., 2000), 13.6\% (Basnet at al., 2009), and 69.7\% (Ho \& Bo, 2006), respectively.

The increasing demand for blood and blood products together with rising costs is a major health care problem (Silberstein et al., 1989). Previous studies have indicated gross over ordering of blood much in excess of actual needs (Friedman et al., 1976). Many units of blood routinely ordered by surgeons are not utilized but are held in reserve and thus are unavailable for other needy patients. This can impose inventory problems for blood bank, loss of shelf life and wastage of blood (Vibhute et al., 2000). On the other hand, with the increased awareness of human immunodeficiency virus infection and other blood-borne infections, blood transfusion has become an issue of increasing concern to both the general public and health practitioners (Friedman et al., 1976).

The preoperative request of blood units, especially in elective surgery, is often based on the worst case assumptions, demanding large quantities of blood or overestimating the anticipated blood loss, of which little is ultimately used (Vibhute et al., 2000). This may cause exhaustion of valuable supplies and resources both in technician time, effort, and biochemical reagents. It also adds to financial burden for each patient undergoing a surgical procedure (Vibhute et al., 2000). Several indices are used to determine the efficiency of blood ordering and utilization system. The cross-match to transfusion ratio (C/T ratio) (Friedman et al., 1976) is the most common index used for evaluating blood transfusion practice. Ideally, this ratio should be 1.0 , but a ratio of 2.5 and below has been suggested to be indicative of efficient blood usage (Ho $\&$ Bo, 2006).The probability of a transfusion for a given procedure is denoted by \% $\mathrm{T}$ as suggested by Mead et al. (1980). A value of 30\% and above has been suggested as appropriate (Ho \& Bo, 2006). The average number of units used per patient cross-match is indicated by the transfusion index ( $\mathrm{TI})$ and signifies the appropriateness of number of units cross-matched. A value of 0.5 or more is indicative of efficient blood usage (Vibhute et al., 2000; Ho \& Bo, 2006). Unnecessary ordering of blood for surgical patients can be reduced without having any detrimental effect on the quality of patient.

Use of blood conservation policies such as the Maximal Surgical Blood Order Schedule (MSBOS) has succeeded in limiting unnecessary transfusion practices (Friedman et al., 1976; Thabah et al., 2013). MSBOS (=1.5 $\times$ TI) estimates the amount of blood that will be needed for the individual procedure. This is a criterion developed from institutional usage statistics providing a figure for the number of units to be cross-matched for any given surgical procedure (Thabah et al., 2013). In the surgeries which have insignificant blood loss, only blood grouping of the patient should be done and cross-matching can be avoided which can not only be rational and cost effective but also hasten the time lost in waiting for surgery. However, one must confirm the availability of blood for emergency situation before starting the surgery (Friedman et al., 1976). Many studies have shown that blood is generally over ordered and the implementation of MSBOS have led to a safe, effective, and economic solution to ordering of blood. Evaluating blood ordering and transfusion practices and subsequent developing of a blood ordering schedule, which serves as a guide to anticipated normal blood usage for elective and emergency surgical procedures, can decrease over-ordering of blood (Vibhute et al., 2000; Mujeeb, 2001).

In most developing countries such as Tanzania, ordering for blood transfusion is frequently based on subjective expectant waiting of blood loss instead of evidence based estimates of average requirement in a particular procedure (Abdelhadi, 2001; Chawla et al., 2001). Many times blood units routinely ordered before elective surgery are not utilized; this imposes storage problems for blood bank, loss of shelf life and wastage of blood (Mahmood et al., 2007). Evidence-based protocols for the transfusion of blood are lacking in most developing countries. Worldwide, the introduction of evidence-based transfusion guidelines and strategies for improved blood utilization has been shown to be cost effective and safe (Kuehn, 2012; Leahy \& 
Mukhtar, 2012; Sherman \& Macivor, 2012). This study was therefore conducted to analyze the pattern of blood requisitions and utilization in the departments of Surgery and Obstetrics/ Gynecology at Bugando Medical Centre in Tanzania. The findings of this study are expected to improve the efficacy of ordering of blood for maximum utilization for commonly performed procedures.

\section{Materials and Methods}

\section{Study design and setting}

This was a retrospective study among patients undergoing major operations in Surgical and Obstetrics and Gynaecology departments of Bugando Medical Centre between April 2013 and March 2014. The study population included all patients undergoing major emergency and elective operations requiring banked homologous blood during the study period. Patients who met the inclusion criteria but who were anaemic had their anaemia corrected before being recruited into the study. Patients who had surgical operations without banked blood or those with no results of cross-matching were excluded from the study.

Based on the nature of surgery performed, the study population was divided into two groups, "elective" and "emergency". At surgery, meticulous dissection and adequate hemostasis with diathermy, where necessary, were employed to minimize blood loss. Need for intraoperative transfusion was determined by amount of blood in soaked gauze swabs, abdominal packs, suction bottle as well as the clinical state of the patient.

The study patients were grouped under separate procedures. Under each procedure the number of patients, units of blood cross-matched and number of units transfused were recorded and the following indices were calculated for each procedure: a) Cross-match to Transfusion ratio $(C / T$ ratio $)=$ Number of units cross-matched $/$ number of units transfused; $b)$ Transfusion Probability $(\% \mathrm{~T})=$ Number of patients transfused $\times 100 /$ number of patients cross-matched; $c$ ) Transfusion Index $(\mathrm{TI})=$ Number of units transfused/ Number of patients cross-matched; d) MSBOS $=1.5 \times$ TI. Adequate blood utilization was defined as a Cross-match (C) to Transfusion ( $\mathrm{T}$ ), C/T ratio of < 2.5 (Boral \& Henry, 1977), Transfusion Index (TI) of 0.5 (Shaikh et al., 2011) and Transfusion Probability (\%T) of $>30$.

The following data were collected - age of patients, sex, diagnosis, nature of surgery, amount of blood loss, amount of blood cross matched, transfused or returned and the reasons for return.

\section{Data analysis}

The data were entered in a pre-designed proforma. Statistical data analysis was done using SPSS software version 17.0 (SPSS, Inc, Chicago, IL). Data was summarized in form of proportions and frequent tables for categorical variables. Continuous variables were summarized using ranges, median and inter-quartile ranges (IQR). The Chi-square $\left(x_{2}\right)$ test was used to test for the significance of association between the independent (predictor) and dependent (outcome) variables in the categorical variables. The level of significance was considered as $p<0.05$. Multivariate logistic regression analysis was used to determine predictor variables that predicted the outcome

\section{Ethical considerations}

Ethical approval to conduct the study was obtained from the Catholic University of Health and Allied Sciences-Bugando/Bugando Medical Centre joint institutional ethic review committee before the commencement of the study.

\section{Results}


During the study period, blood requisition was made to 1,382patients undergoing major surgical procedures. Of this, 588 (42.5\%) were emergency and 794 (57.5\%) were elective operations. The age of patients ranged from 11-78 years with a median of 38 years (+ IQR of 36 and 42 years). Seven hundred eighty-two (56.6\%) were males and 600(43.4\%) were females, giving a male to female ratio of 1.3: 1 .

A total of 1,602 units of blood were cross-matched, out of which $452(28.2 \%)$ units of blood were transfused to 410 (29.7\%) of the patients. This means $71.8 \%$ of units of blood were unutilized. Of the 588 patients who underwent emergency surgery, 690 units of blood were cross matched, out of which only $186(26.9 \%)$ units were transfused to $130(22.1 \%)$ patients, leaving $73.1 \%$ unutilized. Out of 186 units of blood, 141 units were transfused intraoperatively, 36 units postoperatively and the remaining 9 units were transfused preoperatively. Table 1 shows comparison of blood cross matched and transfused in the emergency surgeries.

Table 1: Comparison of blood cross matched and transfused in emergency surgeries at Bugando Medical Centre

\begin{tabular}{|c|c|c|c|c|c|c|c|}
\hline \multirow[b]{2}{*}{ Diagnosis(operation) } & \multicolumn{2}{|c|}{ Cross-matched } & \multicolumn{4}{|c|}{ Transfused } & \multirow[b]{2}{*}{ Total } \\
\hline & Patients & Units & Patients & Preop & Intraop & Postop & \\
\hline Appendicitis (appendicectomy) & 76 & 80 & 1 & 0 & 2 & 0 & 2 \\
\hline Bowel perforation (laparotomy) & 58 & 60 & 10 & 2 & 8 & 2 & 12 \\
\hline Intestinal obstruction (laparotomy) & 55 & 62 & 4 & 0 & 8 & 0 & 8 \\
\hline Peritonitis (laparotomy) & 46 & 52 & 5 & 0 & 5 & 2 & 7 \\
\hline $\begin{array}{l}\text { Open wounds/fractures(surgical } \\
\text { debridement ) }\end{array}$ & 45 & 60 & 22 & 2 & 20 & 4 & 26 \\
\hline $\begin{array}{l}\text { Ruptured ectopic } \\
\text { pregnancy(laparotomy) }\end{array}$ & 42 & 48 & 20 & 2 & 24 & 2 & 26 \\
\hline $\begin{array}{l}\text { Crushed /gangrenous limb(limb } \\
\text { amputation) }\end{array}$ & 40 & 48 & 10 & 3 & 5 & 6 & 14 \\
\hline Splenic injury (splenectomy) & 38 & 48 & 23 & 2 & 20 & 5 & 27 \\
\hline $\begin{array}{l}\text { Obstructed hernias } \\
\text { (herniorrhaphy/Mayo's repair) }\end{array}$ & 38 & 40 & 4 & 0 & 6 & 2 & 8 \\
\hline $\begin{array}{l}\text { Postpartum haemorrhage } \\
\text { (evacuation) }\end{array}$ & 35 & 46 & 14 & 0 & 20 & 6 & 26 \\
\hline $\begin{array}{l}\text { Obstructed labour (Cesarean } \\
\text { section) }\end{array}$ & 32 & 43 & 6 & 0 & 8 & 4 & 12 \\
\hline $\begin{array}{l}\text { Testicular torsion } \\
\text { (orchidopexy/orchidectomy) }\end{array}$ & 29 & 30 & 1 & 0 & 1 & 0 & 1 \\
\hline Head injuries (craniotomy) & 28 & 34 & 6 & 0 & 6 & 1 & 7 \\
\hline Pelvic abscess (laparotomy) & 18 & 33 & 3 & 0 & 7 & 2 & 9 \\
\hline Abortion (evacuation) & 8 & 6 & 1 & 0 & 1 & 0 & 1 \\
\hline
\end{tabular}

Key: intra=intraoperatively; postop= postoperatively; Preop= preoperatively

Generally, the overall blood transfusion of the requested blood as indicated by indices of $\mathrm{C} / \mathrm{T}$ ratio, \% T, TI and MSBOS were 3.5, 28.7\%, 0.33 and 0.45 , respectively. The overall CT ratio, \%T, TI and MSBOS in the emergency operations were $3.7,22 \%, 0.32$ and 0.48 , respectively. On calculation of the blood transfusion indices in each operation, surgical debridement for open wounds/fractures, laparotomy for ruptured ectopic pregnancy, postpartum hemorrhage and splenectomy showed significant blood utilization in all the four indices, while transfusion index was not significant in the rest of other operations (Table 2).

Table 2: Blood transfusion indices in emergency surgeries at Bugando Medical Centre

\begin{tabular}{lllll}
\hline Diagnosis (operation) & CT & \%T & Ti & MSBOS \\
\hline Appendicitis (appendicectomy) & 40.0 & 1.3 & 0.03 & 0.05 \\
\hline 4 & 4 & & &
\end{tabular}




\begin{tabular}{|c|c|c|c|c|}
\hline Bowel perforation (laparotomy) & 5.0 & 17.2 & 0.20 & 0.30 \\
\hline Intestinal obstruction (laparotomy) & 7.8 & 7.2 & 0.15 & 0.23 \\
\hline Peritonitis (laparotomy) & 7.4 & 10.9 & 0.15 & 0.23 \\
\hline Open wounds/fractures(surgical debridement ) & 2.3 & 48.9 & 0.58 & 0.87 \\
\hline Ruptured ectopic pregnancy (laparotomy) & 1.8 & 48.6 & 0.62 & 0.93 \\
\hline Crushed /gangrenous limb(limb amputation) & $3 \cdot 3$ & 25.0 & 0.35 & 0.53 \\
\hline Splenic injury (splenectomy) & 1.8 & 60.5 & 0.71 & 1.07 \\
\hline Obstructed hernias (herniorrhaphy/Mayo's repair) & 5.0 & 10.5 & 0.21 & 0.32 \\
\hline Postpartum haemorrhage (evacuation) & 1.7 & 40.0 & 0.74 & 1.11 \\
\hline Obstructed labour (cesarean section) & 3.6 & 18.8 & 0.38 & 0.57 \\
\hline Testicular torsion (orchidopexy/orchidectomy) & 30.0 & 3.4 & 0.03 & 0.05 \\
\hline Head injuries (craniotomy) & 4.9 & 21.4 & 0.28 & 0.42 \\
\hline Pelvic abscess (laparotomy) & 3.7 & 16.7 & 0.5 & 0.75 \\
\hline Abortion (evacuation) & 3.0 & 12.5 & 0.13 & 0.20 \\
\hline
\end{tabular}

Key: $\mathrm{CT}=$ Cross-match to Transfusion, \% $=$ Transfusion Probability, $\mathrm{Tl}=$ Transfusion Index, MSBOS = Maximal Surgical Blood Order Schedule

Similarly, 912 (56.9\%) units of blood were cross matched for 794 (57.5\%) patients undergoing elective surgery, out of which only $156(17.1 \%)$ units were transfused to $126(15.9 \%)$ patients (i.e. only $17.1 \%$ of total blood cross matched was utilized, leaving $82.9 \%$ unutilized). Of the 156 units, 125 units were transfused intraoperatively, 19 units postoperatively and the remaining 12 units were transfused preoperatively. There was a statistically significant difference between the percentages of blood utilized in emergency operations compared to that in elective operations (26.9\% versus $17.1 \%)(p=0.011)$. Table 3 shows comparison of blood cross matched and transfused in the elective surgeries.

Table 3: Comparison of blood cross matched and transfused in the elective surgeries at Bugando Medical Centre

\begin{tabular}{|c|c|c|c|c|c|c|c|}
\hline \multirow[b]{2}{*}{ Diagnosis(operation) } & \multicolumn{2}{|c|}{ Cross-matched } & \multicolumn{4}{|c|}{ Transfused } & \multirow[b]{2}{*}{ Total } \\
\hline & Patients & Units & Patients & Preop & Intraop & Postop & \\
\hline Goitre (Thyroidectomy) & 86 & 98 & 3 & 0 & 2 & 0 & 2 \\
\hline Abdominal masses (laparotomy) & 80 & 82 & 30 & 4 & 35 & 4 & 43 \\
\hline BPH (prostatectomy/ TURP) & 78 & 89 & 6 & 2 & 4 & 2 & 8 \\
\hline Uterine fibroid (TAH) & 72 & 98 & 6 & 0 & 10 & 0 & 10 \\
\hline $\begin{array}{l}\text { Urethral stricture } \\
\text { (DVU/urethroplasty) }\end{array}$ & 69 & 74 & 0 & 0 & 0 & 0 & 0 \\
\hline Limb fractures (ORIF/ EFX) & 68 & 75 & 7 & 0 & 10 & 0 & 10 \\
\hline Breast cancer (mastectomy) & 65 & 86 & 6 & 1 & 7 & 0 & 8 \\
\hline $\begin{array}{l}\text { Cholelithiasis/ cholecystitis (open } \\
\text { cholecystectomy) }\end{array}$ & 54 & 59 & 0 & 0 & 0 & 0 & 0 \\
\hline $\begin{array}{l}\text { Gastric/bowel cancers } \\
\text { (laparotomy +resection) }\end{array}$ & 50 & 56 & 23 & 2 & 20 & 4 & 26 \\
\hline Splenic tumor (splenectomy) & 37 & 42 & 25 & 2 & 14 & 6 & 22 \\
\hline $\begin{array}{l}\text { Gastric outlet obstruction } \\
\text { (gastrectomy/gastrojejunostomy) }\end{array}$ & 35 & 47 & 12 & 1 & 10 & 2 & 13 \\
\hline $\begin{array}{l}\text { Granulated ulcers/burns (skin } \\
\text { grafting) }\end{array}$ & 33 & 41 & 6 & 0 & 6 & 0 & 6 \\
\hline $\begin{array}{l}\text { Tubo-ovarian masses } \\
\text { (laparotomy) }\end{array}$ & 33 & 46 & 0 & 0 & 0 & 0 & 0 \\
\hline Urolithiasis (urolithotomy) & 18 & 23 & 0 & 0 & 0 & 0 & 0 \\
\hline $\begin{array}{l}\text { Renal masses/non-functioning } \\
\text { kidney (nephrectomy) }\end{array}$ & 12 & 18 & 2 & 0 & 7 & 1 & 8 \\
\hline $\begin{array}{l}\text { Empyema thoracis } \\
\text { (decortications) }\end{array}$ & 4 & 8 & 0 & 0 & 0 & 0 & 0 \\
\hline
\end{tabular}


Key: $\mathrm{BPH}=$ Benign prostatic hyperplasia, $\mathrm{TURP}=$ Transurethral resection prostatectomy, $\mathrm{DVU}=$ Direct Vision Urethrotomy, $\mathrm{TAH}=$ Total abdominal hysterectomy, ORIF= Open reduction and internal fixation, $\mathrm{EFX}=$ External fixation, Preop= Preoperatively, Intraop = Intraoperative, Postop= Postoperatively.

The overall $\mathrm{CT}$ ratio, \% $\mathrm{T}, \mathrm{Ti}$ and MSBOS in the elective operations were $5.8,15.9 \%, 0.2$ and 0.3 , respectively. On calculation of the transfusion indices in each individual operation, laparotomy for abdominal masses, gastric and bowel resection for gastric and bowel tumors and splenectomy showed significant blood utilization while transfusion index was significant in nephrectomy for renal masses/non-functioning kidneys. There was no transfusion in most of the routine surgeries performed in elective basis (Table 4 ).

Table 4: Blood transfusion indices in elective surgeries at Bugando Medical Centre

\begin{tabular}{lllll}
\hline Diagnosis (operation) & CT & \% & Ti & MSBOS \\
\hline Goitre (Thyroidectomy) & 49 & 3.5 & 0.02 & 0.03 \\
Abdominal masses (laparotomy) & 1.9 & 37.8 & 0.54 & 0.81 \\
BPH (prostatectomy/ TURP) & 11.1 & 7.7 & 0.10 & 0.15 \\
Uterine fibroid (TAH) & 9.8 & 8.3 & 0.14 & 0.21 \\
Urethral stricture (DVU/urethroplasty) & $\infty$ & 0 & 0 & 0 \\
Limb fractures (ORIF/ EFX) & 7.5 & 10.3 & 0.15 & 0.23 \\
Breast cancer (mastectomy) & 10.8 & 9.2 & 0.12 & 0.18 \\
Cholelithiasis/ cholecystitis (open cholecystectomy) & $\infty$ & 0 & 0 & 0 \\
Gastric/bowel cancers (laparotomy +resection) & 2.2 & 46.0 & 0.52 & 0.78 \\
Splenic tumor (splenectomy) & 1.9 & 67.6 & 0.59 & 0.89 \\
Gastric outlet obstruction & 3.6 & 34.3 & 0.37 & 0.56 \\
(gastrectomy/gastrojejunostomy) & & & & 0.27 \\
Granulated ulcers/burns (skin grafting) & 6.8 & 18.8 & 0.18 & 0 \\
Tubo-ovarian masses (laparotomy) & $\infty$ & 0 & 0 & 0.17 \\
Urolithiasis (urolithotomy) & 11.5 & 5.6 & 0.11 & 1.00 \\
Renal masses/non-functioning kidney & 2.3 & 16.7 & 0.67 & 0 \\
(nephrectomy) & & & & 0 \\
Empyema thoracis (decortications) & $\infty$ & 0 & & \\
\hline
\end{tabular}

Key: CT = Cross-match to Transfusion, \%T = Transfusion Probability, $\mathrm{Tl}=$ Transfusion Index, MSBOS = Maximal Surgical Blood Order Schedule, BPH = Benign prostatic hyperplasia, TURP= Transurethral resection prostatectomy, DVU= Direct Vision Urethrotomy, $\mathrm{TAH}=$ Total abdominal hysterectomy, ORIF= Open reduction and internal fixation, EFX= External fixation

\section{Discussion}

Blood and its components play a major role in the resuscitation and management of both elective and emergency surgical patients. Despite this advantage, currently there is a limited supply with increasing demand and underutilization of the requested blood worldwide (Ho \& Bo, 2006). The need for blood in surgical units continues to exceed the volume collected by the transfusion services. Studies have shown that there is frequently a gross over-ordering of blood for surgical intervention, in excess of actual and anticipated needs (Friedman et al., 1976; Ho \& Bo, 2006). This leads to substantial costs and a burden to the transfusion services. In addition, over-ordering leads to the non-availability of cross-matched units while reserved for a specific patient (Vibhute et al., 2000).

In this study, about three quarters of the cross-matched blood was unutilized; a figure which is higher than that reported by Belayneh et al (2013) in Ethiopia. But it was relatively low compared to a study conducted in India, Nepal, and Egypt (74.8\%) (Vibhute et al., 2000; Basnet at al., 2009; Ibrahim et al., 2011). Data from developing countries have shown gross over ordering of blood in $40 \%$ to $70 \%$ of patients transfused (Basnet at al., 2009). Generally the percentage of cross-matched patients receiving transfusion for surgical procedures ranged from 5 to $40 \%$ (Ho \& 
Bo, (2006) as compared to $28.2 \%$ of utilization in our hospital. This might indicate that this malpractice is common in developing countries including Tanzania. The over-ordering of blood in our study was probably because of the fear that blood will not be available, when needed. Therefore, it is essential that the usage of blood and blood products should be rationalized and saved for crisis situations.

The current study has also shown that less than one-fifth of total blood cross matched in elective surgical patients was utilized as compared to about a quarter that was utilized in emergency operations. Similar observation has also been reported by Basnet at al. (2009). This observation indicates that the routine cross-matching of blood in elective surgery is a culture rather than a necessity. Disparities in rates of transfusion in the present study are due to the fact that in comparison with emergency operations there is a great tendency to request more units of blood for elective procedures than what is actually required. This over ordering of blood might be due to subjective over blood loss estimation of a procedure by surgeons which usually explain for the provision of safety measure in the event of excessive unexpected blood loss during surgery. Over ordering of blood for elective surgeries can be decreased by simple means of changing the blood cross matching and ordering schedule depending upon the type of surgery performed.

Several indices have been used to determine the efficiency of blood ordering and utilization system. Cross-match to transfusion $(C / T)$ ratio for evaluating blood transfusion is a common practice (Friedman et al., 1976). Ideally, this ratio should be 1.0, but a ratio of 2.5 and below was suggested to be indicative of efficient blood usage. According to this recommendation, the overall $C / T$ ratio of 3.5 that was reported in the current study was considered to be indicative of inefficient blood usage. This ratio is comparable with the ratio that was reported by a study in Egypt (Ibrahim et al., 2011). This observation indicates over-ordering of blood preoperatively that leads to holding up of blood bank reserve as cross-matched blood is considered reserved blood. Patients who may need blood immediately or with legitimate blood requirements may be deprived of it. This leads to aging of blood units and wastage of blood bank resources. In this study, the overall C/T ratio was significantly higher in elective operations than in emergency operations indicating gross over-ordering of blood for elective surgeries, in excess of the actual and anticipated needs. Auditing the surgical blood ordering practice may improve the efficiency of the use of blood for transfusion and avoid unnecessary ordering of blood.

Mead et al. (1980) suggested the probability of transfusion for a given procedure (\%T), which indicates efficient use of blood. Accordingly, a value of $30 \%$ and above has been suggested to be appropriate and signifies the appropriateness of number of units cross matched (Mead et al., 1980). The results of the present study revealed an overall \%T of $28.7 \%$, which was indicative of inappropriate utilization compared to unit cross-matched. This finding was higher than that reported in Indian tertiary care hospital (Vibhute et al., 2000). Similarly, the probability of transfusion reported in elective and emergency operations in the current study both showed inefficient utilization. Regarding transfusion index ( $\mathrm{TI})$, a value of 0.5 or more is indicative of efficient blood usage and signifies the appropriateness of number of units transfused (Friedman et al., 1976). Transfusion index (TI) of both elective and emergency patients this study was considered inappropriate. This finding was lower than the one reported from studies in India and Egypt (Vibhute et al., 2000; Ibrahim et al., 2011). Blood ordering pattern needs to be revised and over ordering of blood should be minimized. This can be possible by the estimation of MSBOS for each procedure and requisition as calculated. Many studies (Vibhute et al., 2000; Mujeeb, 2001) have shown that blood is generally over ordered and the implementation of MSBOS have led to a safe, effective, and economic solution to ordering of blood.

In the current study, significant blood utilization using all four indices in each emergency operation was obtained in surgical debridement for open wounds/fractures, laparotomy for ruptured ectopic pregnancy, postpartum hemorrhage and splenectomy. In elective surgery, laparotomy for abdominal masses, gastric and bowel resection for gastric and bowel tumors, 
splenectomy and nephrectomy for renal masses/non-functioning kidneys showed significant blood utilization. Similar observation was also reported by other authors (Soomro et al., 2011; Ibrahim et al., 2011).

In conclusion, preoperative over-ordering of blood for surgical interventions, in excess of the actual and anticipated needs is a common practice in Bugando Medical Centre. Therefore blood ordering pattern needs to be revised and over ordering of blood should be minimized. This can be possible by the estimation of MSBOS for each procedure and requisition as calculated. In the surgeries which have insignificant blood loss, only blood grouping of the patient should be done and cross matching can be avoided which can not only be rational and cost effective, but also hasten the time lost in waiting for surgery. However, one must confirm the availability of blood for emergency situation before starting the surgery.

\section{References}

Abdelhadi, M. (2001) Blood Conservation in Elective Surgery. Kuwait Medical Journal 33, 232-234. Basnet, R.B., Lamichhane, B. \& Sharma, V.K. (2009) A study of blood requisition and transfusion practice in surgery at Bir Hospital. Postgraduate Medical Journal of NAMS 9, 14-19.

Belayneh, T., Messele, G., Abdissa, Z. \& Tegene, B. (2013) Blood requisition and utilization practice in surgical patients at University of Gondar Hospital, Northwest Ethiopia. Journal of Blood Transfusion http://dx.doi.org/10.1155/2013/758910

Boral, L.I. \& Henry, J.B. (1977) The type and screen: a safe alternative and supplement in selected surgical procedures. Transfusion 17, 163-168.

Chawla, T., Kakepoto, G. \& Khan, M. (2001) An audit of blood cross-match ordering practices at the Aga Khan University Hospital: first step towards a Maximum Surgical Blood Ordering Schedule. Journal of Pakistan Med Association 51, 251-254.

Efraim, K. (2001) Blood conservation in South Africa. A vital need. Bloodless Medicine and Surgery 43, 7-9.

Friedman, B.A., Oberman, H.A., Chadwick, A.R. \& Kingon, K.I. (1976) The maximum surgical blood order schedule and surgical blood use in the United States. Transfusion 380-387.

Hebert, P.C., Wells, G., Blajchman, M.A., Marshall, J., Martin, C., Pagliarello, G., Tweeddale, M., Schweitzer, I. \& Yetisir, E. (1999) A multicenter, randomized, controlled clinical trial of transfusion requirements in critical care. Transfusion Requirements in Critical Care Investigators, Canadian Critical Care Trials Group. New England Journal of Medicine 340, 409417.

Ho, O. \& Bo, B. (2006) Blood utilization in elective surgical procedures in Ilorin. Tropical Journal of Health Sciences 13, 15-17.

Ibrahim, S.Z., Mamdouh H.M., Ramadan M.A. (2011) Blood utilization for elective surgeries at main University Hospital in Alexandria, Egypt. Journal of American Science 7, 683-689.

Kuehn, B.M. (2012) Guidelines tighten transfusion criteria. Journal of the American Medical Association 307, 1788-1789.

Leahy, M.F. \& Mukhtar, S.A. (2012) From blood transfusion to patient blood management: a new paradigm for patient care and cost assessment of blood transfusion practice. International Medical Journal 42, 332-338.

Mahmood, M., Masood J., Shams N.A., Sadia, S.S. \& Manzar, S. (2007) Utilization of blood in Elective Cholecystectomy. Pakistan Journal of Medical Science 23, 331-333.

Mead, J.H., Anthony, C.D. \& Sattler, M. (1980) Haemotherapy in elective surgery: an incidence report, review of literature and alternatives for guideline appraisal. American Journal Clinical Pathology 74, 223-227. 
Mujeeb, S.A. (2001) An audit of blood cross-match ordering practices at the Aga Khan University Hospital: first step towards a maximum surgical blood ordering schedule (MSBOS). Journal of the Pakistan Medical Association 51, 10, 379-380.

Rund, R.L., Bird A.R. \& James M.F.M. (1992) Blood usage in elective surgery: a 3-month audit at Groote Schuur hospital, Cape Town. South African Medical Journal 81, 415-418.

Shaikh, I.A., Umer, M.F. \& Mehdi, H. (2011) Routine cross-match ordering practices, an unnecessary step in routine cholecystectomy. Pakistan Journal of Surgery 27, 271-273.

Sherman, C.H., Macivor, B.C. (2012) Blood utilization: fostering an effective hospital transfusion culture. Journal of Clinical Anesthesia 24, 155-163.

Silberstein, L.E., Kruskall, M.S., Stehling, L.C. Johnston, M.F.M., Rutman, R.C., Samia, C.T. \& Ramsey G. (1989) Strategies for the review of transfusion practices. Journal of the American Medical Association 262, $1993-1997$.

Soomro, R., Javed, M.R \&, Ali, S.A. (2011) Arrangements and use of blood in elective surgical procedures. Professional Medical Journal 18, 212-214.

Sowayan, S. A. (1994) Use of blood in elective surgery: an area of wasted hospital resource. Annals of Saudi Medicine 14, 326-328

Thabah, R., Sailo, L.T., Bardoloi, J., Lanleila, M., Lyngdoh, N.M., Yunus, M. \& Bhattacharyya, P. (2013) Maximum Surgical Blood Order Schedule' in a newly set-up tertiary care hospital. Anaesthesia, Pain \& Intensive Care 17, 28-32.

Vibhute, M., Kamath, S.K. \& Shetty, M. (2000) Blood utilization in elective general surgery cases: requirements, ordering and transfusion practices. Journal of Postgraduate Medicine, 46, 1317. 